\title{
Differences in normal values for murine white blood cell counts and other hematological parameters based on sampling site
}

\author{
J.A. Nemzek, G. L. Bolgos, B. A. Williams and D. G. Remick \\ Department of Pathology, University of Michigan, M2210 Medical Science I, 1301 Catherine Road, Ann Arbor, Michigan 48109-0602, USA, \\ Fax: ++1 734763 6476, e-mail: remickd@umich.edu \\ Received 8 December 2000; returned for revision 27 June 2001; accepted by L. G. Letts 5 July 2001
}

\begin{abstract}
Objective and design: The effect of blood sampling site on the hemogram and neutrophil adhesion molecules was examined in $\mathrm{BALB} / \mathrm{c}$ mice.

Materials and methods: Blood samples were drawn from the tail, eye, and heart during anesthesia with ketamine and xylazine. Cell numbers were quantified with an automated counter and flow cytometry was used to quantify CD11b and CD18.

Results: Total white blood cell (WBC) counts were highest from tail, lower from eye, and significantly lower from heart blood. In general, differences between tail and heart counts reflected changes in all cell types. RBCs, platelets and hematocrits were significantly increased in tail compared to heart blood. Although CD18 levels were not different, CD11b was significantly higher on neutrophils from tail compared to heart blood.

Conclusions: In anesthetized BALB/c mice, sampling site readily influences blood counts and neutrophil CD11b. The findings underscore the need to standardize sampling site when measuring these parameters.
\end{abstract}

Key words: WBC counts - Differentials - CD11b - RBC count

\section{Introduction}

In studies of complex inflammatory responses, the evaluation of multiple, interrelated parameters including peripheral blood cell counts, WBC differentials, and the presence of cell adhesion molecules has become an integral part of many in vivo experiments. In the past, repeated evaluation of these parameters in murine models was hampered by the limited blood volume of the subjects and the labor involved in processing large numbers of the samples. However, technical advances including automated cell counters and flow cytometry now allow rapid evaluation of complete blood counts [1, 2] and other factors from very small amounts of blood.

Correspondence to: D. G. Remick
Although the methods to quantify cell numbers are readily available, the investigator must be aware that intrinsic variability may arise in cell counts in some species. It has been reported that white blood cell counts and differentials can vary substantially between strains, sexes, and ages of mice [3], illustrating the importance of consistency during an experiment. Of even greater significance, the white blood cell counts from an individual mouse, under certain circumstances, can also vary greatly when the blood is obtained from different sampling sites. It has been established that the total WBC counts obtained from the heart blood of unanesthetized mice and of rats anesthetized with Nembutal are significantly lower than those from tail [4] or retroorbital blood [5]. However, these differences do not appear in rodents that are anesthetized with ether $[4,6]$. The effects of sample site on cell counts in mice anesthetized with other anesthetic agents such as ketamine and xylazine have not been evaluated.

As with cell counts, quantification of cell adhesion molecules may be important to the evaluation of inflammatory responses but also can be subject to variability. Several factors are known to modulate the presence of the $\beta$-integrins CD11b and CD18 including cytokines, endotoxin [7], oxygen radicals [8], purification techniques [9], and anesthetic agents $[10,11]$. However, it is unknown if the presence of neutrophil adhesion molecules varies between sample sites, as do the white blood cell counts.

The classic studies examining the effects of sample sites were performed a number of years ago on several different strains of mice without the benefit of automated cell counters or currently used anesthetic regimens. In order to appropriately control our own in vivo murine studies, we sought to further investigate the effects of blood sample site on frequently measured parameters of inflammation. The first aim of this study was to examine the effects of sampling site (heart, tail, eye) and order on the hemogram of a commonly used strain of mouse, the BALB/c, and to quantify these effects with an automated system. The second aim was to determine the effects of sampling site and order on the presence of the integrins CD11b and CD18 on neutrophils in these mice. Finally, we would determine if the effects seen in 
these mice, anesthetized with ketamine and xylazine, would be comparable to the historical data taken from mice restrained by other means.

\section{Materials and methods}

\section{Study design}

With institutional animal care and use approval, blood samples were drawn from anesthetized mice at three sampling sites, tail, eye, and heart. In Group $1(n=6)$, the order of blood sampling sites used was tail, eye, heart. In Group $2(n=6)$, the order of sampling was eye, tail, heart. Because the heart blood collection was a terminal procedure and last in the order of sampling in these two groups, a third group of mice $(n=3)$ was used for sampling of heart blood only.

\section{Animals}

Female, BALB/c mice (Charles River Laboratories, Wilmington, MA) weighing 19-20 grams were used for sample collection. The animals were kept under standard laboratory conditions with a 12:12 hour dark/light cycle for at least one week prior to sample collection.

\section{Anesthesia}

Animals were anesthetized with ketamine $(83 \mu \mathrm{g} / \mathrm{g})$ and xylazine $(13 \mu \mathrm{g} / \mathrm{g})$ administered intraperitoneally for all of the blood drawing procedures including tail blood samples.

\section{Blood collection}

Tail: The distal one-half centimeter of the tail was clipped and a capillary pipette containing anticoagulants (EDTA for cell counting, heparin for flow cytometry) were used to collect two $20 \mu \mathrm{l}$ samples from the bleeding surface. The first sample was used for cell counting and the second for flow cytometry. Immediately after collection, the cut surface of the tail was cauterized with styptic powder (Kwik-stop, ARC Laboratories, Atlanta, GA) [12].

Eye: As previously described [12], a capillary pipette containing anticoagulant (EDTA for cell counting, heparin for flow cytometry) was inserted in the lateral canthus and blood collected from the retroorbital sinus. A total of $40 \mu \mathrm{l}$ of blood was removed form this site. After collection, the pipette was removed and bleeding stopped when the eye returned to a normal position.

Heart: With the animal positioned on its back, the abdomen was opened and the heart visualized through the membranous part of the diaphragm. A 21 gauge needle and $3 \mathrm{cc}$ syringe were used to obtain $0.5 \mathrm{ml}$ of blood from the left ventricle. The blood was then immediately transferred to two $20 \mu \mathrm{l}$ capillary pipettes containing the appropriate anticoagulants for cell counting and flow cytometry.

\section{Sample processing}

Blood counts: EDTA anti-coagulated blood samples were used to obtain a complete blood count with a Hemavet Mascot Multispecies Hematology System Counter 1500R (CDC Technologies, Inc., Oxford, CT). Samples were counted no longer than five minutes after blood was drawn.

Flow cytometry: A $20 \mu \mathrm{l}$ aliquot of heparinized blood was mixed with phycoerythrin-labeled anti-CD11b and fluorescein isothiocyanatelabeled anti-CD18 (Pharmingen, San Diego, CA). The samples were incubated on ice for one half-hour. Flow cytometry was then immedi- ately performed by the core facility at the University of Michigan, Ann Arbor, MI. The results were expressed as mean fluorescence intensity of neutrophils.

\section{Statistics}

Summary statistics were expressed as the mean \pm SEM. The differences between sample sites were compared with an ANOVA. Tukey's test for pairwise comparisons was performed when indicated. Student's t-test was used to compare the order of sample collection from a particular sampling site between different individuals.

\section{Results}

Since the heart blood samples were obtained last in the series in Groups 1 and 2, these samples were compared to values from heart blood obtained without prior bleeding. There was no significant difference based on the order of blood draw in these samples. Therefore, the two groups were combined for further comparison between sampling sites.

The cell counts obtained from the first blood sample drawn in each group were compared (Fig. 1). Overall, the
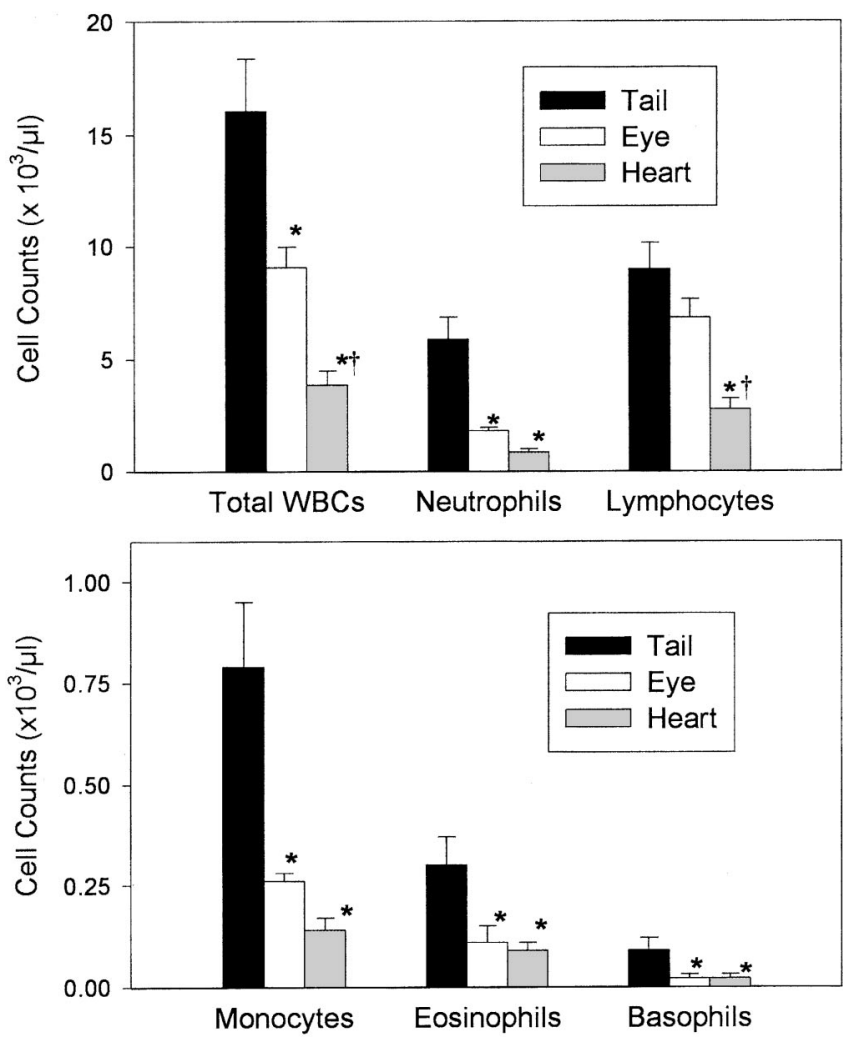

Fig. 1. Effect of Sample Site on WBC Counts. BALB/c mice were anesthetized with ketamine and xylazine. Blood was drawn from either the tail $(n=6)$, eye $(n=5)$, or heart $(n=15)$ and cells were counted with an automated counter. Total WBC counts were highest in blood taken from the tail and significantly lower in blood from the heart. The lower counts reflected decreases in all of the types of WBCs. Cell counts from the eye were, in general, intermediate when compared to the other sites. Values are expressed as the mean \pm SEM. ${ }^{*}=\mathrm{p}<0.05$ as compared to the Tail group. $\dagger=\mathrm{p}<0.05$ as compared to the Eye group. 
Table 1. Hematological data taken from unanesthetized, adult female BALB/c Mice*.

\begin{tabular}{lll}
\hline Parameter & Mean \pm SEM & Range \\
\hline Total WBCs $\left(\times 10^{3} / \mathrm{ul}\right)$ & $17.7 \pm 1.034$ & $9.1-28.7$ \\
Neutrophils $\left(\times 10^{3} / \mathrm{ul}\right)$ & $5.7 \pm 0.4$ & $1.9-11.5$ \\
Lymphocytes $\left(\times 10^{3} / \mathrm{ul}\right)$ & $10.5 \pm 0.5$ & $6.7-15.7$ \\
Monocytes $\left(\times 10^{3} / \mathrm{ul}\right)$ & $0.7 \pm 0.06$ & $0.3-1.4$ \\
Eosinophils $\left(\times 10^{3} / \mathrm{ul}\right)$ & $0.2 \pm 0.03$ & $0.05-0.51$ \\
Platelets $\left(\times 10^{3} / \mathrm{ul}\right)$ & $934.7 \pm 34.5$ & $668-1543$ \\
Hematocrit $(\%)$ & $51.2 \pm 1.0$ & $39.7-71.8$ \\
RBC $\left(\times 10^{6} / \mathrm{ul}\right)$ & $10.2 \pm 0.2$ & $8.48-15.15$ \\
MCV $(\mathrm{fL})$ & $50.7 \pm 0.4$ & $47.4-54.4$ \\
Hemoglobin $(\mathrm{g} / \mathrm{dL})$ & $16.9 \pm 0.2$ & $14.8-18.3$ \\
\hline
\end{tabular}

* Blood obtained from the tail and analyzed by Hemavet ${ }^{\circledR}$.

WBC counts obtained from tail blood samples were higher than those from either heart or eye and were within the ranges previously obtained in our laboratory from 24, unanesthetized BALB/c mice (Table 1). The differences in the total WBC counts between the tail samples and either the eye or heart samples were significant. The lower total WBC counts in the eye samples reflected significant changes in the numbers of neutrophils, monocytes, eosinophils, and basophils compared to the tail samples. The lymphocyte counts were not significantly different between the tail and eye samples. The even lower WBC counts obtained from heart samples represented significant decreases in lymphocyte numbers as well as neutrophils and the other WBC types when compared to the tail samples. Although all of the types of the WBCs were generally lowest in the heart samples, the significant difference in total WBC counts between the eye and heart samples were primarily due to a difference in the lymphocyte counts.

As with the WBC counts, the tail blood samples demonstrated higher amounts of CD11b than did eye samples and the heart samples demonstrated the least amount of CD11b (Fig. 2). There was a significant difference in mean fluores-

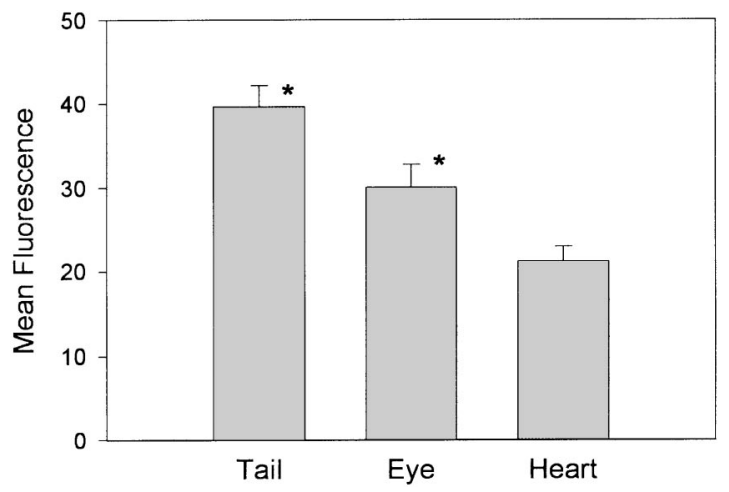

Fig. 2. Effects of Sample Site on CD11b. BALB/c mice were anesthetized with ketamine and xylazine. Blood was drawn from either the tail $(n=6)$, eye $(n=6)$, or heart $(n=15)$ and cells were labeled with pycoerythrin-labeled anti-CD11b for flow cytometry. Fluorescence was significantly increased on neutrophils obtained from tail and eye blood samples as compared to the heart blood samples. ${ }^{*}=\mathrm{p}<0.05$ as compared to the Heart group.
Table 2. Hematological data from blood samples drawn from three different sites.

\begin{tabular}{llll}
\hline Parameter & Tail Blood & Eye Blood & Heart Blood \\
\hline Platelets $\left(\times 10^{3} / \mathrm{ul}\right)$ & $882 \pm 95$ & $637 \pm 56$ & $344 \pm 73^{*}$ \\
Hematocrit $(\%)$ & $53.15 \pm 2.27$ & $46.12 \pm 1.90^{*}$ & $41.16 \pm 1.11^{*}$ \\
RBC $\left(\times 10^{6} / \mathrm{ul}\right)$ & $10.94 \pm 0.54$ & $10.14 \pm 0.42$ & $9.00 \pm 0.22^{*}$ \\
MCV $(\mathrm{fL})$ & $48.85 \pm 1.94$ & $45.5 \pm 0.62$ & $45.5 \pm 0.33$ \\
Hemoglobin $(\mathrm{g} / \mathrm{dL})$ & $15.5 \pm 1.15$ & $14.28 \pm 0.59$ & $13.04 \pm 0.34$ \\
\hline
\end{tabular}

$*=\mathrm{p}<0.05$ as compared to the tail blood sample; $\dagger=\mathrm{p}<0.05$ as compared to eye blood samples.

cence between the tail and heart blood samples. The amount of CD18 did not differ between the sampling sites.

In addition to the WBC parameters, several other factors were examined (Table 2). Platelet counts were significantly higher in tail blood samples when compared to heart blood. The hematocrit was significantly higher in the tail blood than in the heart blood samples. This was probably due to a significant decrease in RBC count in the heart blood samples since the hematocrit presented by the automated counter is a value derived from the total RBC count and the mean corpuscular volume (MCV). Hemoglobin values were not different between the groups.
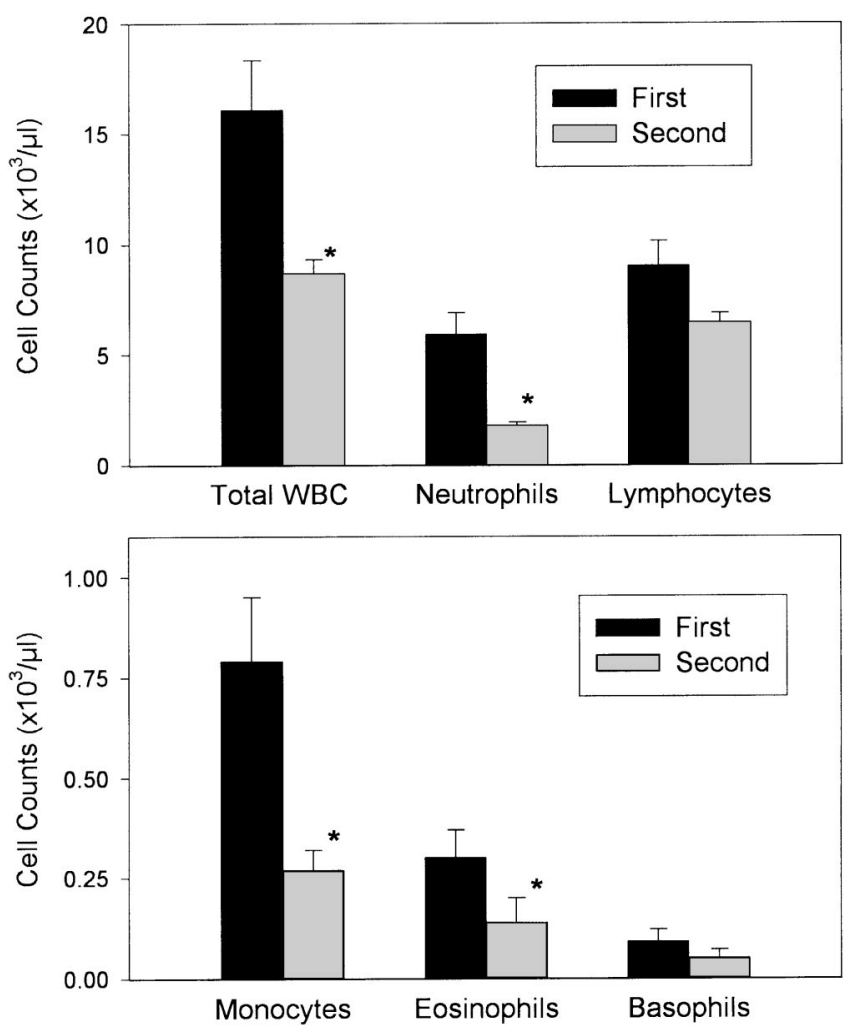

Fig. 3. Effect of Sample Order on WBC Counts. BALB/c mice were anesthetized with ketamine and xylazine. Blood was drawn from the tail either before (First, $\mathrm{n}=6$ ) or after (Second, $\mathrm{n}=5$ ) a sample was taken from the eye and cells were counted with an automated counter. Total WBC counts were significantly higher in the blood taken before additional sampling. The change in total WBC count reflected changes in neutrophil, monocyte, and eosinophil counts. Values are expressed as the mean \pm SEM. $*=p<0.05$. 
Table 3. Hematological data from tail blood samples drawn either before (first) or after (second) eye samples.

\begin{tabular}{lll}
\hline Parameter & First & Second \\
\hline Platelets $\left(\times 10^{3} / \mathrm{ul}\right)$ & $882 \pm 95$ & $563 \pm 69^{*}$ \\
Hematocrit $(\%)$ & $53.15 \pm 2.27$ & $44.5 \pm 2.29^{*}$ \\
RBC $\left(\times 10^{6} / \mathrm{ul}\right)$ & $10.94 \pm 0.54$ & $9.65 \pm 0.05$ \\
MCV $(\mathrm{fL})$ & $48.85 \pm 1.94$ & $46.15 \pm 0.43$ \\
Hemoglobin $(\mathrm{g} / \mathrm{dL})$ & $15.5 \pm 1.15$ & $12.84 \pm 0.59$ \\
\hline
\end{tabular}

$*=\mathrm{p}<0.05$

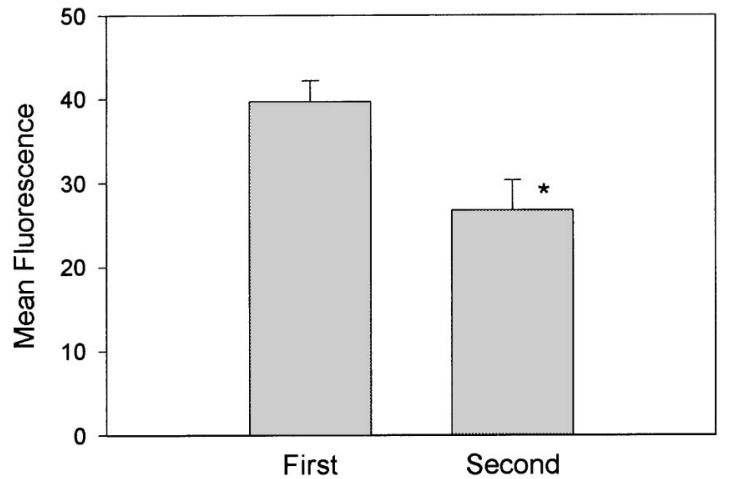

Fig. 4. Effects of Sample Order on CD11b. BALB/c mice were anesthetized with ketamine and xylazine. Blood was drawn from the tail either before (First, $\mathrm{n}=6$ ) or after (Second, $\mathrm{n}=6$ ) a sample was taken from the eye and cells were labeled with phycoerythrin-labeled anti$\mathrm{CD} 11 \mathrm{~b}$ for flow cytometry. Fluorescence was significantly higher in the samples taken prior to additional sampling. ${ }^{*}=\mathrm{p}<0.05$. All values are expressed as the mean \pm SEM.

The order of blood draw (either first or second) from the tail and the eye was also evaluated. There were no significant differences in any of the cell counts or the mean fluorescence of the neutrophil adhesion molecules when the samples were taken from the eye first (Group 2) or taken from the eye after the tail sample (Group 1). In contrast, the tail samples taken first (Group 1) demonstrated a significantly higher WBC count when compared to tail samples drawn after eye samples (Group 2) (Fig. 3). These counts reflected significant differences in neutrophil, monocyte, and eosinophil counts. The platelet counts and hematocrit of samples taken from the tail were higher when the sample was drawn before the eye sample than when drawn after the eye sample (Table 3). In addition, the mean fluorescence of $\mathrm{CD} 11 \mathrm{~b}$ was significantly higher in the tail blood samples drawn first (Fig. 4).

\section{Discussion}

The published studies examining the effects of sample site on cell counts were conducted decades ago on several different strains of mice under various anesthetic protocols and produced variable results. Our study updates this information by using an automated cell counter to obtain information from a commonly used strain of mouse subjected to a frequently used anesthetic protocol.
The results of this study demonstrated consistently that cell counts are highest in tail blood, lower in eye blood and lowest in the heart. These results are similar to the results of other studies of blood obtained from rodents. Sakaki [5] reported that RBC counts and WBC counts were significantly higher in blood obtained from the tail of mice when compared to that taken from the eye. Although the percentage of neutrophils and lymphocytes found in the eye samples was often higher than those in the tail samples, the absolute numbers of neutrophils and lymphocytes was not reported in that study. Goldie [13] examined murine blood obtained from several sites from the tail tip to the heart and found that WBC counts progressively decreased from the periphery to the heart. Likewise, Quimby $[4,14]$ showed dramatic decreases in WBC counts in samples taken from the heart versus the tail of rats. In one of those studies, no differences in WBC differential counts in rats were seen when samples from the heart and tail were compared [4]. In addition, that study did not demonstrate an effect due to the order of sampling. The results of differential counts and order of sampling in that study contradicted the findings of our study but may be due to variation in species or anesthetic protocols.

While the reason for the differences seen in the WBC counts may be rooted in the immunological demands of the host at the various sites [14], the physiological explanation for the differences has not been completely defined. It has been suggested that the vascular resistance and blood stasis created by the larger surface area in the capillary beds may be responsible for the higher WBC counts from peripheral sites [4]. This theory is supported by the fact that anesthesia with ether equalized the counts in the tail and heart due to its vasodilatory effects $[4,6]$. However, the "damming up" that is believed to occur in the capillary beds of the tail [4] was not evident upon histological evaluation of tail sections taken from mice in our study (data not shown). In addition, anticoagulation with heparin will also equalize the blood counts without inducing vasomotor affects [13]. Alternatively, it has been suggested that there is a fluid exchange between the vessels and tissues of the tail in rodents [13].

Since $\mathrm{CD} 11 \mathrm{~b}$ also varied with sample site, we considered the possibility that it was responsible for the changes in WBC counts. The amount of CD11b was lowest in the heart blood samples as were the total WBC counts, suggesting that the neutrophils with the higher CD11b levels could have adhered and essentially been filtered out by the lung capillary bed. However, this would not completely explain the effects of sample site on the WBC count since all types of WBCs and RBCs were lower in heart blood than in tail blood, suggesting a flow mechanics mechanism. It is important to note that the differences in CD11b were detectable in the face of ketamine anesthesia, known to attenuate the upregulation of $\mathrm{CD} 11 \mathrm{~b}$ [11].

In conclusion, this study demonstrated that sampling site can profoundly influence the total and differential white blood cell, RBC, and platelet counts obtained from BALB/c mice under ketamine and xylazine anesthesia. The order that is used to draw samples from the tail can also influence these parameters. Both sampling site and the order of tail blood draw will also affect the amount of CD11b found on neutrophils. The findings emphasize the need to standardize sampling site and order when measuring these parameters in research studies. 


\section{References}

[1] Ebong SJ, Call DR, Bolgos G, Newcomb DE, Granger JL, O'Reilly $\mathrm{M}$, et al. Immunopathologic responses to non-lethal sepsis. Shock 1999; 12: 118-26.

[2] Nemzek JA, Call DR, Ebong SJ, Newcomb DE, Bolgos GL, Remick DG. Immunopathology of a two-hit murine model of acid aspiration lung injury. Am J Physiol; Lung Cell Mol Physiol 2000; 278: L512-L520.

[3] Bannerman RM. Hematology. In: Foster HL, Small JD, Fox JG, editors. The Mouse in Biomedical Research. vol. 3. New York, N.Y.: Academic Press, 1983: 293-309.

[4] Quimby FH, Goff LG. Effect of source of blood sample on total white cell count of the rat. Am J Physiol 1952; 170: 196-200.

[5] Sakaki K, Tanaka K, Hirasawa K. Hematological comparison of the mouse blood taken from the eye and the tail. Exper Anim 1961; 10: 14-9.

[6] Nichols J, Miller AT. A comparison of the total leucocyte count in the heart blood and peripheral blood of the rat. Science 1948; 108: 378-9.

[7] McMillen MA, Huribal M, Sumpio B. Common pathway of endothelial-leukocyte interaction in shock, ischemia, and reperfusion. Am J Surg 1993; 166: 557-62.
[8] Fraticelli A, Serrano CV, Jr, Bochner BS, Capogrossi MC, Zweier JL. Hydrogen peroxide and superoxide modulate leukocyte adhesion molecule expression and leukocyte endothelial adhesion. Biochim Biophys Acta 1996; 1310: 251-9.

[9] Kuijpers TW, Tool AT, van der Schoot CE, et al. Membrane surface antigen expression on neutrophils: a reappraisal of the use of surface markers for neutrophil activation. Blood 1991; 78: 1105-11.

[10] Schmidt H, Ebeling D, Bauer H, et al. Ketamine attenuates endotoxin-induced leukocyte adherence in rat mesenteric venules. Crit Care Med 1995; 23: 2008-14.

[11] Weigand MA, Schmidt H, Zhao Q, Plaschke K, Martin E, Bardenheuer HJ. Ketamine modulates the stimulated adhesion molecule expression on human neutrophils in vitro. Anesth Analg 2000; 90: 206-12.

[12] Cuncliffe-Beamer TL. Biomethodology and surgical techniques. In: Foster HL, Small JD, Fox JG, editors. The Mouse in Biomedical Research. vol. 3. New York, N.Y.: Academic Press, 1983: 402-37.

[13] Goldie H, Jones AM, Ryan H, Simpson M. Leucocyte counts in the blood from the tail and heart of the mouse. Science 1954; 119: 353-4.

[14] Quimby FH, Saxon PA, Goff LG. Total white blood cell counts of peripheral and heart blood samples of the rat. Science 1948; 107: 447.

\section{(17) To access this journal online:

